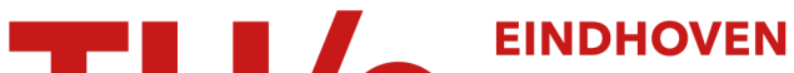 \\ UNIVERSITY OF \\ TECHNOLOGY
}

\section{Towards plasmonic lasers for optical interconnects}

Citation for published version (APA):

Dolores Calzadilla, V. M., Fiore, A., \& Smit, M. K. (2012). Towards plasmonic lasers for optical interconnects. In Proceedings of the 2012 14th International Conference on Transparent Optical Networks (ICTON), 2-5 July 2012, Warwick, United Kingdom (pp. Th.A5.7-1/4). Institute of Electrical and Electronics Engineers.

https://doi.org/10.1109/ICTON.2012.6254472

DOI:

10.1109/ICTON.2012.6254472

Document status and date:

Published: 01/01/2012

\section{Document Version:}

Publisher's PDF, also known as Version of Record (includes final page, issue and volume numbers)

\section{Please check the document version of this publication:}

- A submitted manuscript is the version of the article upon submission and before peer-review. There can be important differences between the submitted version and the official published version of record. People interested in the research are advised to contact the author for the final version of the publication, or visit the $\mathrm{DOI}$ to the publisher's website.

- The final author version and the galley proof are versions of the publication after peer review.

- The final published version features the final layout of the paper including the volume, issue and page numbers.

Link to publication

\section{General rights}

Copyright and moral rights for the publications made accessible in the public portal are retained by the authors and/or other copyright owners and it is a condition of accessing publications that users recognise and abide by the legal requirements associated with these rights.

- Users may download and print one copy of any publication from the public portal for the purpose of private study or research.

- You may not further distribute the material or use it for any profit-making activity or commercial gain

- You may freely distribute the URL identifying the publication in the public portal.

If the publication is distributed under the terms of Article $25 \mathrm{fa}$ of the Dutch Copyright Act, indicated by the "Taverne" license above, please follow below link for the End User Agreement:

www.tue.nl/taverne

Take down policy

If you believe that this document breaches copyright please contact us at:

openaccess@tue.nl

providing details and we will investigate your claim. 


\title{
Towards Plasmonic Lasers for Optical Interconnects
}

\author{
V. Dolores-Calzadilla ${ }^{1}$, A. Fiore ${ }^{2}$, M. K. Smit ${ }^{1}$ \\ COBRA Research Institute, Eindhoven University of Technology, The Netherlands \\ ${ }^{I}$ Photonic Integration, Department of Electrical Engineering \\ ${ }^{2}$ Photonics and Semiconductor Nanophysics, Department of Applied Physics \\ E-mail:V.Calzadilla@tue.nl
}

\begin{abstract}
A plasmonic laser structure coupled to a dielectric waveguide is proposed and investigated by rigorous simulations. Modal characteristics, such as propagation loss and confinement factor were obtained for a wavelength of $1.55 \mu \mathrm{m}$. In addition, FDTD (Finite-Difference Time-Domain) simulations were used to calculate the reflectivity from the end facets of the laser structure and also to calculate the coupling between the laser and a dielectric waveguide. Simulations show that a threshold gain of $1796 \mathrm{~cm}^{-1}$ is required to compensate the total loss in a $200 \mathrm{~nm}$ wide and $50 \mu \mathrm{m}$ long laser, which can be achievable at room temperature.
\end{abstract}

Keywords: plasmonic lasers, photonic integrated circuits, optical interconnects, plasmonics, nanophotonics.

\section{INTRODUCTION}

During the last two decades, the research on photonic integrated circuits has shown a progressive development leading to an increase in complexity and functionality. An important development is the combination of silicon with III-V materials to create efficient light sources. Different technologies are under investigation, IMOS (InP Membrane On Silicon) being a promising approach for the implementation of active components [1]. This technology aims for InP-based photonic circuits in a membrane bonded to silicon by means of BCB.

Optical interconnects are of special interest because they are currently a promising alternative to replace electrical interconnects in order to solve the problem of continuously increasing data rates [2]. Furthermore, the use of optical interconnects would allow for devices with ultra-low power consumption, nanometric footprint and reduced crosstalk by the use of metallic encapsulation [2], [3].

For the realization of optical interconnects, a number of devices have to be designed and integrated. In this contribution, a plasmonic laser coupled to a low-loss dielectric waveguide is proposed. It has an additional quaternary layer on top, which is required for reasons discussed below. Such a bilayer dielectric waveguide can easily be converted to a single-layer waveguide as used in the IMOS-technology, by tapering horizontally the quaternary layer to push down the optical mode [4].

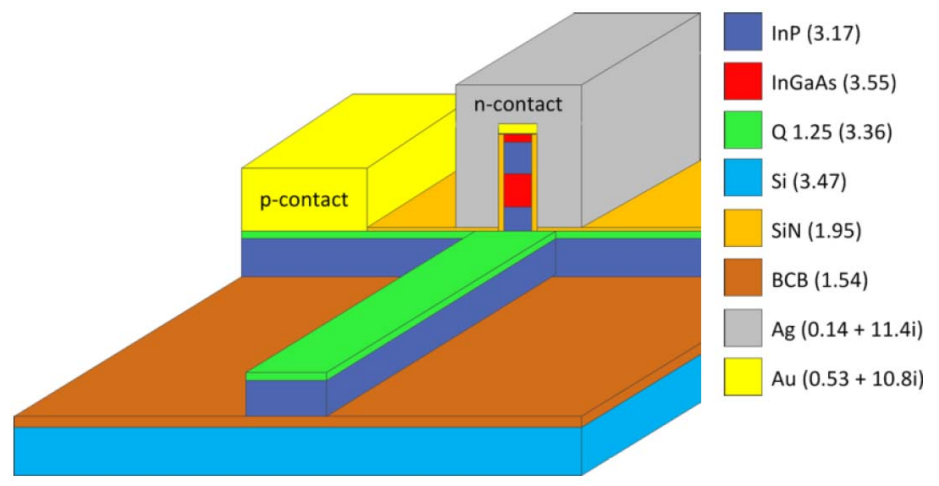

Figure 1. Plasmonic laser coupled to a dielectric waveguide. The refractive index of each material at $1.55 \mu \mathrm{m}$ is shown in parenthesis. Optical absorption in InGaAs has been neglected for the simulations.

The laser structure proposed is shown in Fig. 1 and it is based on previously reported plasmonic lasers able to lase at cryogenic temperatures [5]. The laser consists fundamentally of a MISIM (metal-insulatorsemiconductor-insulator-metal) waveguide forming a Fabry Perot resonator, on an InP-membrane, coupled to a dielectric waveguide. The top n-contact and the lateral p-type contact should provide the electrical pumping for the InGaAs active medium, which has a bandgap at $1.65 \mu \mathrm{m}$.

As it can be seen in Fig. 1, there is a thin insulating layer of SiN between the semiconductor layer stack and the metal cladding, which serves to insulate the structure horizontally and therefore allow a top-down current flow. The quaternary (Q 1.25) layer acts as the ohmic contact layer for the p-contact. The back side of the Fabry Perot cavity is completely terminated by metal in order to achieve a strong reflection, whereas it has an open facet at the frontal end to achieve the outcoupling. For the simulations, the frequency dependent refractive indices of the InP-lattice-matched materials were described with a modified single oscillator model [6], whereas gold and silver were described with experimental data [7]. 


\section{MODAL PROPERTIES}

The intensity distribution of the hybrid surface plasmon polariton mode with lowest loss in the cavity is shown in Fig. 2b. Due to its plasmonic nature, it has a dominant $E_{x}$ component and is mainly confined within the insulation layer, which leads to a very poor overlap with the active region. The post height $h$ is important because it will determine the reflectivity and outcoupling coefficient.
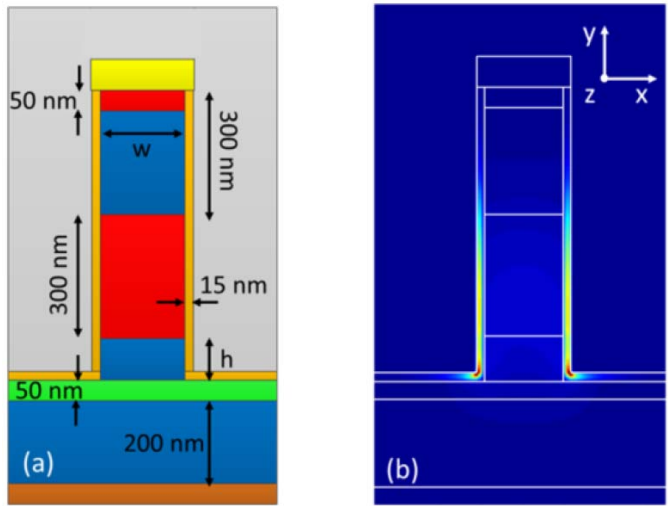

Figure 2: (a) Cross section of the plasmonic laser cavity with the dimensions used for the simulations;

(b) Optical intensity of the plasmonic mode at $1.55 \mu \mathrm{m}$. Blue: low intensity. Red: high intensity.

There are two main problems to deal with for the design of a plasmonic laser of this kind. The first one is the high propagating loss due to the large metal absorption, and the second one is the low confinement factor of the plasmonic mode supported by the MISIM structure. These two factors are responsible for the very high threshold gain required, as it is discussed below.

The propagation loss as a function of the width $w$, can be observed in Fig. 3 for different values of the post height. This loss is due to the absorption by the metal and it decreases when the core is wider because the mode is increasingly confined in the core.

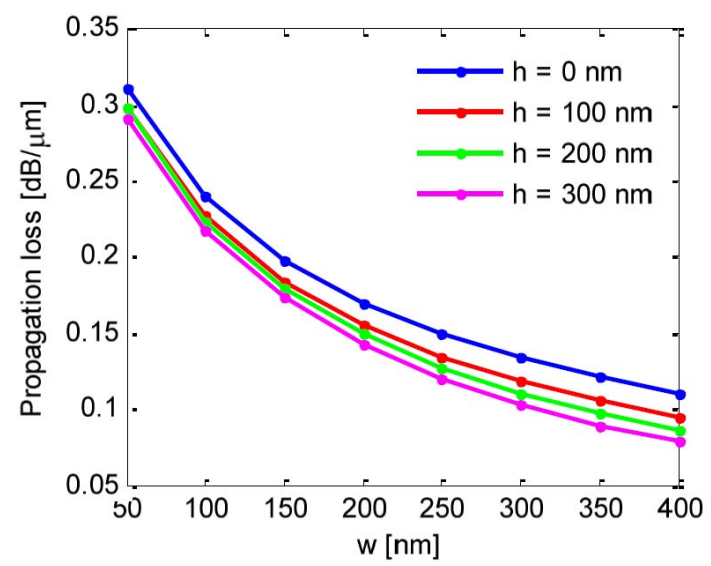

Figure 3. Propagation loss due to the optical absorption of the metal cladding for a wavelength of $1.55 \mu \mathrm{m}$.

An increase in the width of the MISIM structure also leads to a higher confinement factor as it is shown in Fig. 4a. The confinement factor is defined as the ratio of the electric field square amplitude within the active medium with respect to the square of the total electric field amplitude and therefore determines the modal gain.

The post height $h$ has also an influence on the loss and the confinement factor due to the presence of the $\mathrm{BCB}$ substrate, which has a lower refractive index than the active medium and therefore contributes to the confinement in the active region. For this reason, when the post height is increased, the mode extends more in the InP post and both the modal loss and the confinement in the active region decrease. The confinement factor is low because the plasmonic mode has its maximum at the interface between the metal and the dielectric as seen in Fig. 2b. The confinement into the active region can be increased by either using an insulation layer with higher refractive index or decreasing the thickness of this layer [8]. For example, the intensity cross section along the core for different thicknesses of the insulation layer is shown in Fig. 4b. As it can be seen, the intensity within the core drops significantly when including the insulation layer, and it decreases slowly when its thickness is increased. However, the loss also decreases for increasing thicknesses of the insulation layer. 

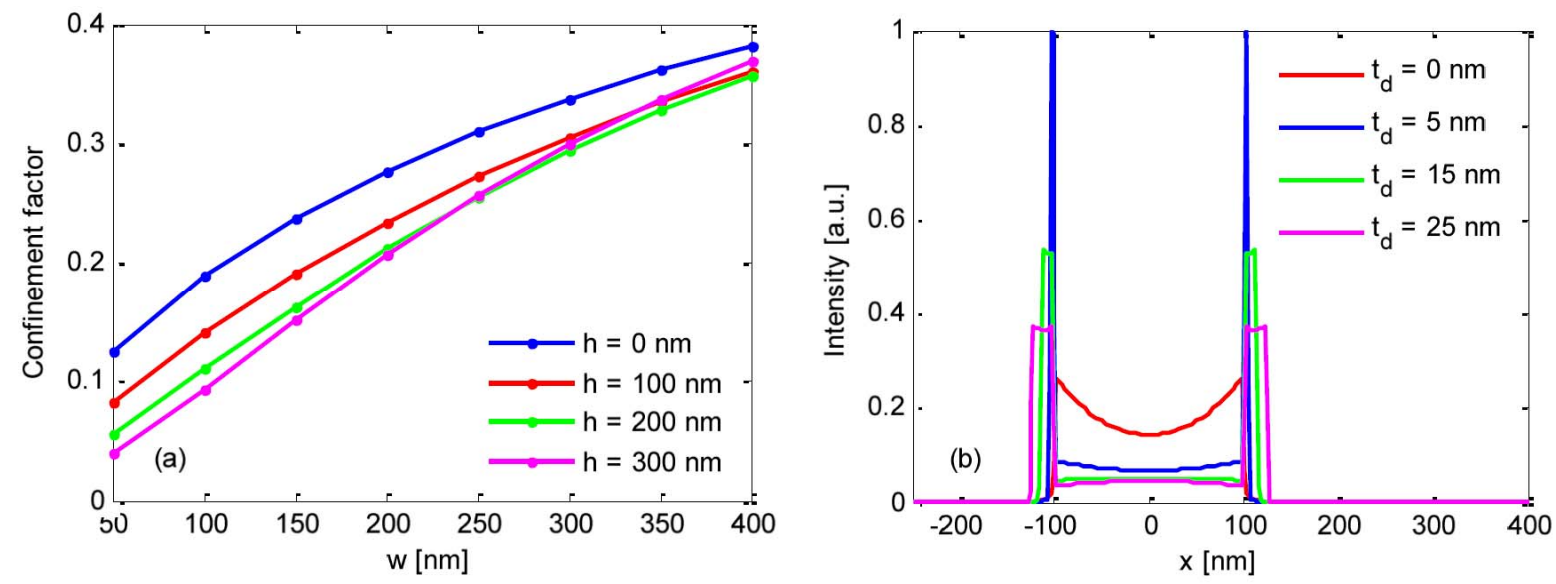

Figure 4: (a) Confinement factor as a function of the MISIM width for several values of the post height, for a wavelength of $1.55 \mu \mathrm{m}$; (b) Normalized intensity along the core for different thicknesses $t_{d}$ of the insulation layer, using $h=0 \mathrm{~nm}$.

\section{FACET REFLECTIVITY AND OUTCOUPLING}

The aforementioned structure was investigated with 3D FDTD simulations in order to calculate the reflectivity of its facets and the outcoupling to the dielectric waveguide. The reflectivity is defined as the normalized optical power reflected back into the laser at the output facet, whereas the outcoupling is calculated as the normalized power coupled into the output waveguide.

As it is observed in Fig. 1, the laser is coupled to an IMOS waveguide with a thin InGaAsP layer on top. Even though the laser core and the waveguide are not vertically aligned, a considerable outcoupling coefficient has been obtained as it is described below. This is possible due to the quaternary layer, which has a high refractive index and therefore facilitates the outcoupling.

Figure 5 shows the reflectivity and outcoupling coefficient as a function of wavelength for an open facet and different post heights (colored curves). The first remarkable characteristic is the high reflectivity of around 0.6 at $1.55 \mu \mathrm{m}$, which is twice the Fresnel reflectivity for an interface given by InGaAs and air, considering perpendicular incidence. This strong reflection is due to the large effective mode index mismatch between surface plasmon modes and free propagating modes in air, and it was found to increase even more for very narrow MISIM structures. In addition, the reflectivity at the backside of the laser which is assumed to have a metal coating, is shown in black in Fig. 5a. As expected, it is high and hardly varies for different values of $h$.
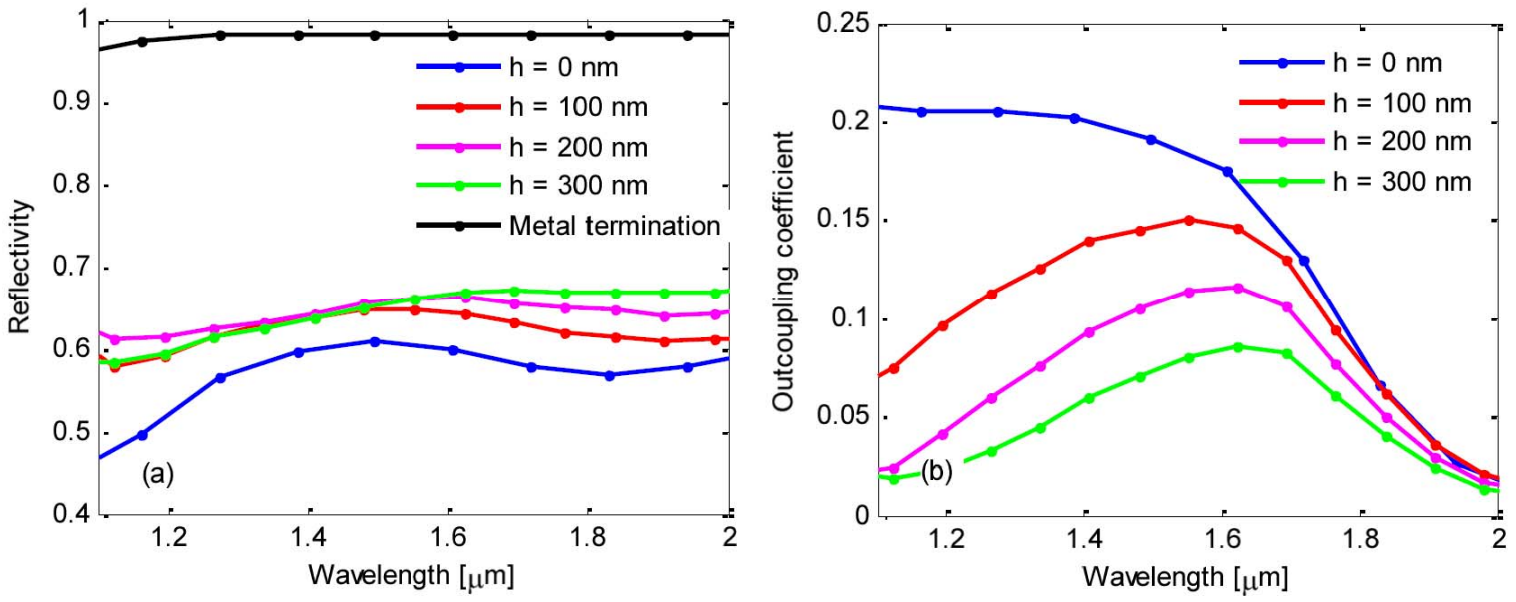

Figure 5: (a) Reflectivity of a laser structure with $w=200 \mathrm{~nm}$, for an open facet with post height $h$ and a metal termination with $h=0 \mathrm{~nm}$; (b) Outcoupling coefficient of a laser structure with $w=200 \mathrm{~nm}$.

The reflectivity presents only a small increase on increasing the post height because it is limited to the reflection between the laser structure and air. On the other hand, the outcoupling continuously decreases and becomes zero for a very high post. Since our main interest is to design an efficient laser, a post height of $100 \mathrm{~nm}$ would be suitable for an operating wavelength of $1.55 \mu \mathrm{m}$, since it maximizes the reflectivity without compromising the outcoupling. Using a higher post will lead to a decrease in the laser efficiency. 
Other solutions can be considered to increase the reflectivity at the outcoupling interface, such as a metallic termination at the output interface. However this would decrease the outcoupling coefficient and lead to excitation of unwanted plasmonic modes at the bottom and corners of the metal termination.

As it is shown in the 2D longitudinal cross section of Fig. 6, the high reflectivity creates a standing wave inside the cavity, whereas a fraction of the transmitted power is coupled into the dielectric waveguide.

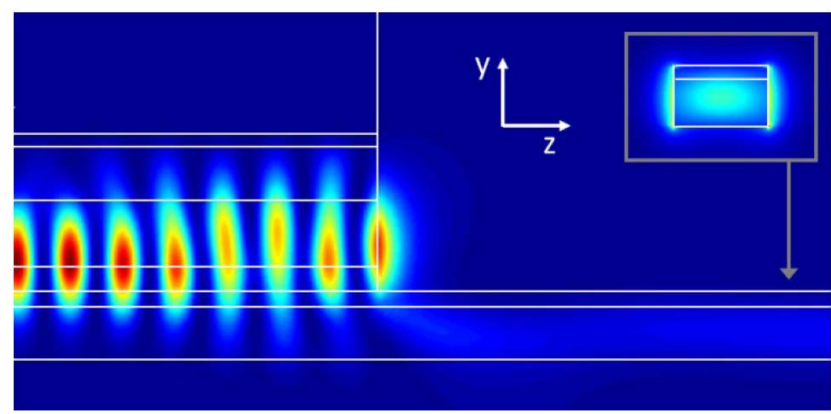

Figure 6. Longitudinal cross section showing the coupling between the laser and the dielectric waveguide. The inset shows a transversal cross section of the waveguide. Blue: low intensity. Red: high intensity.

Finally, the gain required to overcome the total loss can be estimated from the equation

$$
g_{t h} \Gamma=\alpha+\frac{1}{2 L} \ln \left(\frac{1}{R_{1} R_{2}}\right)
$$

where $g_{t h}$ is the material threshold gain, $\Gamma$ is the confinement factor, $\alpha$ is the mode propagation loss, $L$ is the length of the Fabry Perot cavity and $R_{1 / 2}$ are the reflectivities of its facets. For example, assuming $L=50 \mu \mathrm{m}$ and considering a laser cavity with $w=200 \mathrm{~nm}$ and $h=100 \mathrm{~nm}$, it gives $\alpha=0.16 \mathrm{~dB} / \mu \mathrm{m}, \Gamma=0.23$, $R_{1}=0.65$ and $R_{2}=0.98$, resulting in a threshold gain of $g_{t h}=1796 \mathrm{~cm}^{-1}$ with only $11 \%$ of efficiency, which is possible at room temperature under a high injected carrier density above $6 \cdot 10^{18} \mathrm{~cm}^{-3}[9]$. Alternatively, a threshold gain of $g_{t h}=2580 \mathrm{~cm}^{-1}$ and an efficiency of $38 \%$ are obtained when considering $L=10 \mu \mathrm{m}$, however, such a high material gain is expected to be achievable only at cryogenic temperatures.

\section{CONCLUSIONS}

A coupled plasmonic laser device has been proposed and investigated, which aims to be compatible with IMOS technology. Despite the relatively large propagation loss and low confinement factor, this structure is interesting in view of the high reflectivity at its open facet and coupling to a dielectric waveguide mode. The threshold gain can be compatible with room temperature operation, albeit with a poor efficiency and at high carrier densities.

\section{ACKNOWLEDGEMENTS}

This work was supported by the EU FP7 project NAVOLCHI with grant agreement no. 288869.

\section{REFERENCES}

[1] J. van der Tol et al:: Photonic integration in Indium-Phosphide Membranes on Silicon (IMOS), IET Optoelectronics, vol. 5, no. 5, pp. 218-225, 2011.

[2] J. A. Conway et al.: Plasmonic interconnects versus conventional interconnects: A comparison of latency, cross- talk and energy costs, Optics Express, vol. 15, no. 8, pp. 1069-1071, 2007.

[3] H. M. G. Wassel et al.: Towards chip-scale plasmonic interconnects, Workshop on the Interaction between Nanophotonic Devices and Systems (WINDS), pp. 1-2, 2010.

[4] M. Lamponi et al.: Low-threshold heterogeneously integrated InP/SOI lasers with a double adiabatic taper coupler, Photonics Technology Letters, vol. 24, no. 1, pp. 76-78, 2012.

[5] M. T. Hill: Status and prospects for metallic and plasmonic nano-laser, J. Opt. Soc. Am. B, vol. 27, no. 11, pp. 36-44, 2010.

[6] F. Fiedler, A. Schlachetzki: Optical parameters of InP-based waveguides. Solid-State Electronics, vol. 30, no. 1, pp. 73-83, 1987.

[7] P. B. Johnson, R. W Christy: Optical constants of nobel metals, Phys. Rev. B, vol. 6, no. 12, 1972.

[8] M. Marell: PhD thesis: Gap plasmon mode distributed feedback lasers, Technische Universiteit Eindhoven, 2011.

[9] C. Y. Lu, S. L. Chuang: A surface-emitting 3D metal-nanocavity laser: Proposal and theory, Optics Express, vol. 19, no. 14, pp. 13225-44, 2011. 